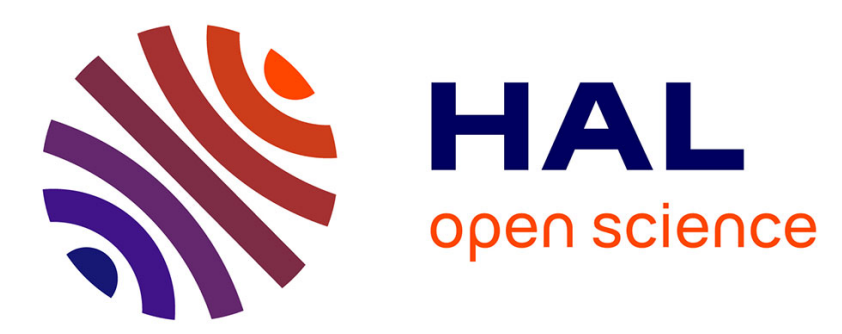

\title{
Insight into electronic mechanisms of nanosecond-laser ablation of silicon
}

\author{
Wladimir Marine, N.M. Bulgakova, Lionel Patrone, Igor Ozerov
}

\section{To cite this version:}

Wladimir Marine, N.M. Bulgakova, Lionel Patrone, Igor Ozerov. Insight into electronic mechanisms of nanosecond-laser ablation of silicon. Journal of Applied Physics, 2008, 103, pp.094902. 10.1063/1.2903527 . hal-00303806

\section{HAL Id: hal-00303806 https://hal.science/hal-00303806}

Submitted on 19 Oct 2017

HAL is a multi-disciplinary open access archive for the deposit and dissemination of scientific research documents, whether they are published or not. The documents may come from teaching and research institutions in France or abroad, or from public or private research centers.
L'archive ouverte pluridisciplinaire HAL, est destinée au dépôt et à la diffusion de documents scientifiques de niveau recherche, publiés ou non, émanant des établissements d'enseignement et de recherche français ou étrangers, des laboratoires publics ou privés. 


\title{
Insight into electronic mechanisms of nanosecond-laser ablation of silicon
}

\author{
Wladimir Marine, ${ }^{1}$ Nadezhda M. Bulgakova, ${ }^{2, a)}$ Lionel Patrone, ${ }^{1, b)}$ and Igor Ozerov ${ }^{1}$ \\ ${ }^{1}$ Centre de Recherche en Matière Condensée et Nanosciences, UPR 7251 CNRS Université de la \\ Méditerranée, Case 913, 163 Avenue de Luminy, 13288 Marseille Cedex 9, France \\ ${ }^{2}$ Institute of Thermophysics SB RAS, 1 Lavrentyev Ave., 630090 Novosibirsk, Russia
}

(Received 14 October 2007; accepted 4 February 2008; published online 1 May 2008)

\begin{abstract}
We present experimental and theoretical studies of nanosecond ArF excimer laser desorption and ablation of silicon with insight into material removal mechanisms. The experimental studies involve a comprehensive analysis of the laser-induced plume dynamics and measurements of the charge gained by the target during irradiation time. At low laser fluences, well below the melting threshold, high-energy ions with a narrow energy distribution are observed. When the fluence is increased, a thermal component of the plume is formed superimposing on the nonthermal ions, which are still abundant. The origin of these ions is discussed on the basis of two modeling approaches, thermal and electronic, and we analyze the dynamics of silicon target excitation, heating, melting, and ablation. An electronic model is developed that provides insight into the charge-carrier transport in the target. We demonstrate that, contrary to a commonly accepted opinion, a complete thermalization between the electron and lattice subsystems is not reached during the nanosecond-laser pulse action. Moreover, the charging effects can retard the melting process and have an effect on the overall target behavior and laser-induced plume dynamics. (c) 2008 American Institute of Physics. [DOI: 10.1063/1.2903527]
\end{abstract}

\section{INTRODUCTION}

Photoinduced processes in solids exposed to pulsed laser irradiation are of prime interest for a number of rapidly developing laser-assisted technologies such as pulsed laser deposition, material microprocessing, nanomaterial synthesis, etc. Among the materials widely used in microindustrial applications, silicon and other semiconductors attract special attention due to their remarkable electrical and optical properties. They demonstrate, however, a variety of physical effects that have not been fully understood. There are still debates about ultrafast melting, ${ }^{1-5}$ resolidification dynamics, ${ }^{6-8}$ surface structure modification, ${ }^{9,10}$ thermal and nonthermal mechanisms of ablation, ${ }^{6,11-18}$ and direct cluster emission. ${ }^{16,19-21}$ This stimulates extensive studies, both experimental and theoretical, of the dynamics of laser heating, melting, resolidification, and ablation of silicon under laser irradiation with different pulse durations and laser wavelengths. It has been shown that the silicon ablation mechanisms can be controlled by tailored laser pulses via governing the thermodynamic pathways of material evolution. ${ }^{22,23}$ The dynamics of nanosecond-laser heating and the melting threshold are usually described within the frames of the thermal model based on the heat-flow equation. ${ }^{24,25}$ At shorter laser pulses, the electron and lattice subsystems are treated separately, involving ambipolar diffusion ${ }^{26-29}$ or drift-diffusion ${ }^{14,30,31}$ approaches. By using a drift-diffusion formalism, it has been demonstrated that the macroscopic Coulomb explosion in silicon is improbable at femtosecond,

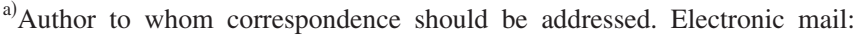
nbul@itp.nsc.ru.

${ }^{b)}$ Present address: Institut Matériaux Microélectronique Nanosciences de Provence (IM2NP) UMR 6242 CNRS, ISEN-Toulon, Maison des Technologies, Place G. Pompidou, F-83000 Toulon, France.
}

IR laser irradiation, ${ }^{30}$ while the conditions for electrostatic disintegration of the external layer of the silicon targets can be created at longer, nanosecond UV pulses ${ }^{31,32}$ with fluences below the melting threshold.

In this paper, we present the results of experimental and theoretical studies of the electron-hole dynamics in a silicon sample irradiated by excimer laser pulses of nanosecond duration. The dynamics of target charging and melting as well as the evolution of the desorption/ablation yields are followed experimentally. It has been shown that, with increasing laser fluence, more energy is required to reach the melting threshold, so that the melting moment is shifted toward the laser pulse tail. An explanation of this effect can be given in terms of energy abstraction due to electronic ablation of superficial layers of the sample and in the redistribution of the electronic population (and hence energy) within the target. In order to reveal the role of electron photoemission and charge-carrier transport in the overall target behavior, we apply two different modeling approaches. The first approach is the conventional thermal model, while the other approach takes into account charging effects in the laser-irradiated samples. ${ }^{32}$ The latter model has been adopted to account for a moving sample boundary due to electronic ablation.

The paper is organized as follows. In Sec. II, we give the details of the experimental procedure and present the experimental results. Section III is devoted to the analysis of the processes that take place in the laser-irradiated semiconductor sample. We describe the thermal model and a model of laser-induced surface charging (the LISC model) specifying electronic ablation. In Sec. IV, the results on modeling obtained by the use of the two different approaches are presented and the processes connected with charge-carrier dynamics are analyzed in detail. 


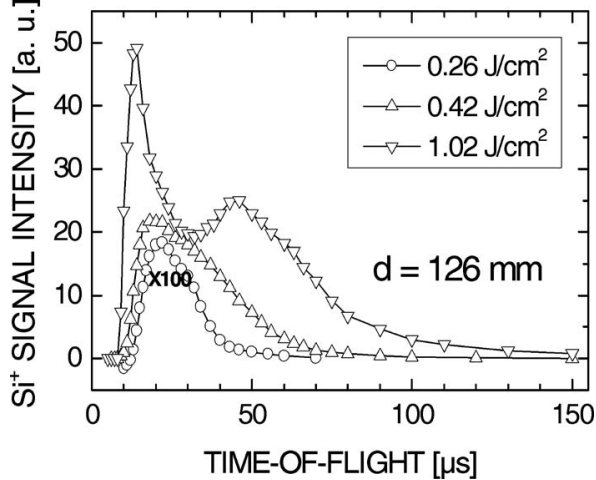

FIG. 1. Time-of-flight spectra of $\mathrm{Si}^{+}$ions measured at different laser fluences. For $0.26 \mathrm{~J} / \mathrm{cm}^{2}$, the vertical scale is expanded by a factor of 100 .

\section{EXPERIMENTAL}

In the experiments, we used a clean (111) Si surface passivated by hydrogen in the usual chemical method. ${ }^{33} \mathrm{Im}$ mediately after treatment, the monocrystalline Si target was introduced into a high vacuum chamber (pressure $<10^{-7}$ Torr) and was irradiated at an angle of incidence of $45^{\circ}$ using an ArF excimer laser $[\hbar \omega=6.4 \mathrm{eV}$ and $15 \mathrm{~ns}$ pulse duration at full width at half maximum (FWHM)]. A set of masks was used to select the homogeneous part of the laser beam. The laser spot size on the target and laser fluence $\left(F_{0}\right)$ were $0.5 \mathrm{~mm}$ and $0.01-1 \mathrm{~J} / \mathrm{cm}^{2}$, respectively. The target was rotated/translated during measurements to avoid cratering. The expansion dynamics and nature of the desorbed particles were analyzed by a reflectron time-of-flight (TOF) mass spectrometer (MS) at a distance of $126 \mathrm{~mm}$ from the target. Typical MS resolution is 500 .

The most abundant desorbed species, monatomic ions $\mathrm{Si}^{+}$, have been observed already at a low laser fluence of about $0.2 \mathrm{~J} / \mathrm{cm}^{2}$, well below the melting threshold $F_{0 \text { th }}$ $=0.4 \mathrm{~J} / \mathrm{cm}^{2}{ }^{34}$ Neutral monatomic Si particles have been detected only at fluences higher than $0.8 \mathrm{~J} / \mathrm{cm}^{2}$. All attempts to detect charged or neutral clusters, except for silicon ion dimers, ${ }^{35}$ have been unsuccessful. The main information concerning expansion dynamics and corresponding ablation mechanisms was obtained from TOF spectra. Figure 1 shows typical TOF spectra of $\mathrm{Si}^{+}$at a fluence well below the melting threshold, also near the beginning of melting, and at a fluence corresponding to the well developed ablation. At low laser fluences $\left(<F_{0 \text { th }}\right)$, the TOF spectra present only one population of $\mathrm{Si}^{+}$ions. The kinetic energy $(\sim 4.8-5 \mathrm{eV})$ and temporal width $(\sim 15 \mu \mathrm{s})$ are very weak functions of laser fluence up to the melting threshold. The spatial distribution of this kind of ions shows a narrow expansion, strongly peaked relative to the target normal. Such a behavior is typical of photoinduced, nonthermal desorption-ablation. ${ }^{36,37}$

The subsequent increase in laser fluence above $F_{0 \text { th }}$ leads to a broadening of the $\mathrm{Si}^{+}$TOF distribution (see Fig. 1, fluence of $0.42 \mathrm{~J} / \mathrm{cm}^{2}$ ) and to the formation of a well pronounced low energy distribution starting from 0.7 to $0.8 \mathrm{~J} / \mathrm{cm}^{2}$ (onset of thermal ablation). The appearance of the second $\mathrm{Si}^{+}$distribution coincides with the development of surface melting. The intensity of this population rapidly increases with fluence and this population is predomi-

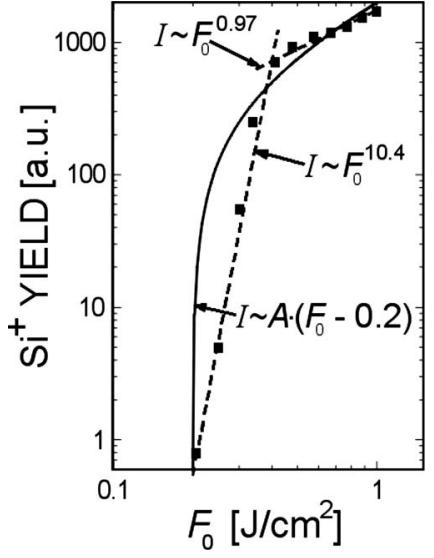

FIG. 2. Experimental data on $\mathrm{Si}^{+}$yield vs laser fluence $F_{0} . F_{0}=0.2 \mathrm{~J} / \mathrm{cm}^{2}$ corresponds to the ion desorption threshold. Up to the melting threshold $\left(\sim 0.4 \mathrm{~J} / \mathrm{cm}^{2}\right)$, the ion yield behaves as $F_{0}^{10.4}$, whereas at higher fluences, the dependence changes to $F_{0}^{0.97}$. The solid curve corresponds to Eq. (27) with $F_{0 \mathrm{cr}}=0.2 \mathrm{~J} / \mathrm{cm}^{2}$.

nant at fluences above $1 \mathrm{~J} / \mathrm{cm}^{2}$. The origin of the second population is clearly determined by thermal phenomena induced by laser irradiation. The analysis of the abundance of $\mathrm{Si}^{+}$species versus laser fluence (Fig. 2, experimental points) clearly shows the transition between the two ejection regimes. The first one, which corresponds to the generation of the fast population and to the nonthermal ablation, exhibits a very strong nonlinear fluence dependence that can be fitted by a $F_{0}^{10.4}$ law. The high order nonlinear dependence is a typical signature of the multiphoton ejection process. ${ }^{36}$ The second regime, which starts after melting, shows a near linear $\mathrm{Si}^{+}$abundance variation versus fluence, thus confirming the thermal nature of the low energy population.

The expulsion of the nonthermal $\mathrm{Si}^{+}$population is induced by the formation of a positive charge on the silicon surface resulting from the emission of photoelectrons during UV laser irradiation. Figure 3 shows a time-integrated laserinduced charge on the target as a function of fluence. This charge has been evaluated by measuring a compensating current variation between the $\mathrm{Si}$ target and the ground ${ }^{38}$ during laser pulse. From this figure, it can be seen that the target already accumulates a positive charge at low laser fluence, $\sim 0.001 \mathrm{~J} / \mathrm{cm}^{2}$. The charge increases almost linearly up to saturation at a fluence of about $0.15-0.2 \mathrm{~J} / \mathrm{cm}^{2}$. Exactly at

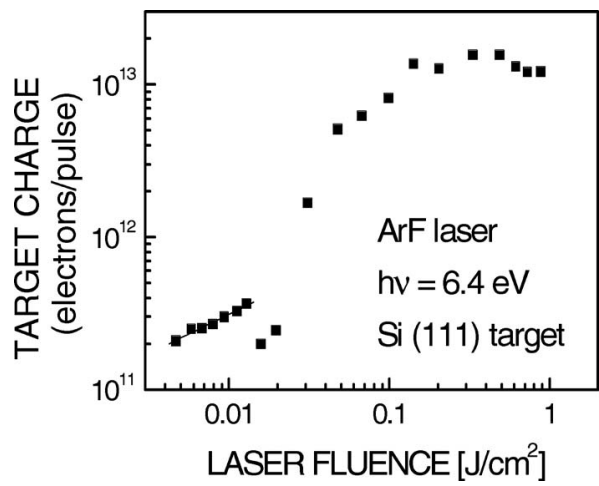

FIG. 3. The measured laser-induced charge of the silicon target vs laser fluence. 


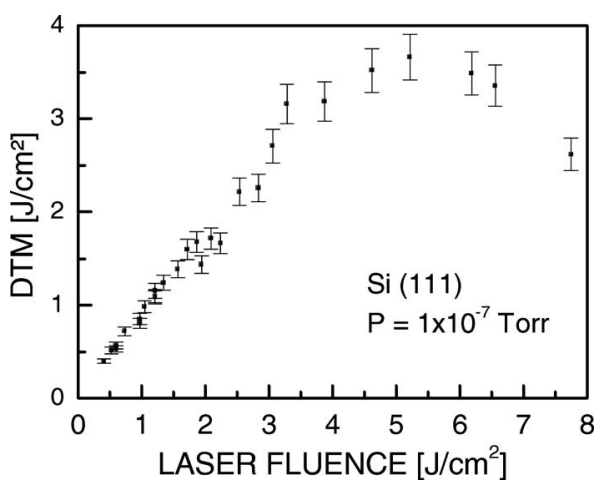

FIG. 4. The DTM measured on a Si(111) surface under vacuum conditions as a function of incident laser fluence. The error bars correspond to the experimental uncertainty on the measurement of the melting moment.

these fluences, we detect the first nonthermal ions. Both the target charge saturation and ion ejection clearly indicate that the Si surface reaches critical conditions corresponding to the dynamical equilibrium between primary particle ejection (photoelectrons) and desorption of the secondary species (positively charged ions).

As a consequence, a part of the absorbed laser energy is used to produce the nonthermal $\mathrm{Si}^{+}$population. This is revealed by evaluating a dynamical threshold of melting (DTM) for various laser fluences using time resolved reflectivity measurements ${ }^{39}$ on a hydrogen passivated $\mathrm{Si}(111)$ surface, under vacuum conditions. In these measurements, a $\mathrm{He}-\mathrm{Ne}$ laser beam is focused on the irradiated Si target area and the temporal evolution of the reflected light is followed. At the instant of surface melting induced by ArF laser irradiation, the reflected He-Ne laser beam intensity jumps to a higher value. Measuring this time from the start of the ArF laser pulse enables us to calculate the necessary fluence to melt a fixed number of Si layers. The obtained results on the DTM are shown in Fig. 4 as a function of ArF laser fluence. The increase in the DTM in the fluence range extending from the minimum static melting threshold of $\sim 0.4 \mathrm{~J} / \mathrm{cm}^{2}$ up to $\sim 5 \mathrm{~J} / \mathrm{cm}^{2}$ is in agreement with the absorption of laser energy by Si surface sites, leading to nonthermal ion ejection. ${ }^{40}$ Although for fluences higher than $\sim 0.4 \mathrm{~J} / \mathrm{cm}^{2}$ the nonthermal population is less pronounced, its yield is still increasing approximately linearly with fluence (see Fig. 2). The DTM decrease for high laser fluences (beyond $\sim 5 \mathrm{~J} / \mathrm{cm}^{2}$ ) was also reported in Ref. 39. It may be explained by the appearance of photoinduced plasma of free carriers, leading to a softening of the crystalline lattice. Then, the material can be in a quasiliquid state even below its melting temperature, ${ }^{41,42}$ which consequently reduces the measured DTM. The difference between the irradiation laser fluence $F_{0}$ and the DTM corresponds to the fluence part directly involved in the target heating beyond melting. This value is plotted versus laser fluence in Fig. 5. It is interesting to note that from the melting threshold $\left(\sim 0.4 \mathrm{~J} / \mathrm{cm}^{2}\right)$ to the thermal ablation threshold $\left(\sim 0.7-0.8 \mathrm{~J} / \mathrm{cm}^{2}\right)$, the $\left(F_{0}-\mathrm{DTM}\right)$ value is close to zero, indicating that the main part of the laser fluence is used in the melting process. Then, beyond the thermal ablation threshold, it starts to grow slowly, transforming to a strong nearly linear increase from a laser fluence of $\sim 3.5 \mathrm{~J} / \mathrm{cm}^{2}$.

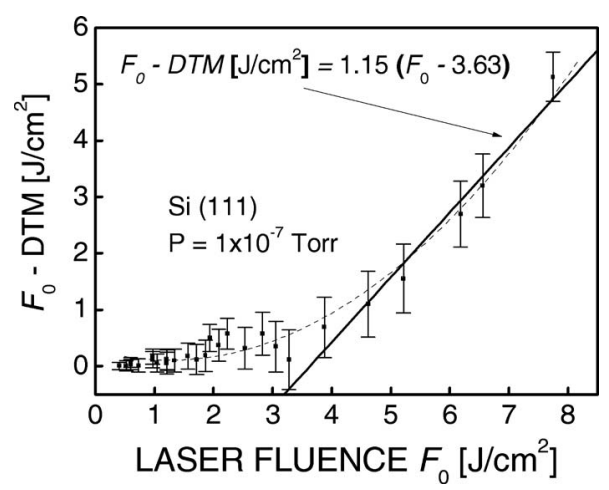

FIG. 5. The difference between incident laser fluence $F_{0}$ and the DTM (the fluence fraction spent for heating the melted $\mathrm{Si}$ surface) as a function of incident laser fluence. The error bars correspond to the experimental uncertainty on the measurement of the melting moment.

Such a behavior with three regimes is consistent with the observations of Sanchez et al. ${ }^{43}$ on the ablation rate of $\mathrm{Si}$ under nanosecond ArF laser irradiation.

\section{MODELING APPROACHES}

The widely used modeling approach for the description of the nanosecond-laser pulse heating and the ablation of materials regardless of their kind is based on the thermal model in its different modifications, including those which take into account irradiation shielding by the generated plasma plume..$^{24,25,44-46}$ This relatively simple model gives a good description of experimental data on the material melting and ablation thresholds and on mass removal without using any adjusting parameters or with a minimum adjustment which, however, can be physically founded. By being incorporated into a number of combined approaches, ${ }^{47-52}$ it has allowed a proper description of the interrelation between the target state and the laser-induced plume behavior including backward deposition of the ablated products. ${ }^{50,52}$ In the model, the absorption of the laser light by the target material is described by the following general Beer-Lambert law, which implies a linear relationship between the absorptance and the concentration of absorbing centers:

$$
I(z)=I_{0} \exp \left(-\alpha_{\mathrm{ab}} z\right),
$$

where $I$ and $I_{0}$ are the local and incident laser intensities, $\alpha_{\mathrm{ab}}$ is the absorption coefficient, and $z$ is the laser beam propagation distance. This implies that the multiphoton processes as well as the free electrons generated in semiconductor and dielectric materials do not play a perceptible role in laser light absorption at the nanosecond regimes of laser irradiation. With the use of a thermal model, the spatial and temporal dynamics of laser heating, melting, and recrystallization can be revealed including the ablation rates via the thermal mechanism and details concerning target superheating. As soon as one is interested in the electronic mechanisms of ablation or in the influence of the free-electron population on the processes taking place in semiconductors or dielectrics under pulsed laser irradiation, a more sophisticated model is required for adequate analysis.

Let us first consider the processes triggered by a nanosecond-laser pulse in a semiconductor target. We will 


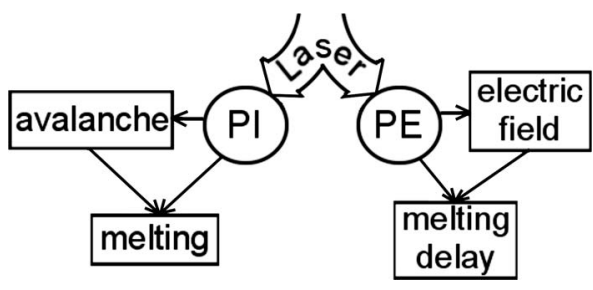

FIG. 6. A simplified scheme of the processes taking place in a silicon target under pulsed laser irradiation demonstrating their impact on the melting onset.

diverge from the assumption of local thermal equilibrium between the electron and lattice subsystems, which is usually implied for nanosecond-laser pulses, and consider the possibility of the violation of target quasineutrality due to electron photoemission. A simplified scheme of the processes is presented in Fig. 6. A laser pulse acting on the target causes photoionization (PI) and photoemission (PE). These processes appear to be competitive with respect to target heating and melting. The electrons excited to the conduction band absorb laser radiation and can produce secondary electrons via collisional multiplication (avalanche). The free electrons, irrespective of their origin, interact with phonons and target heating takes place, which, at a definite level of the absorbed laser energy, gives rise to the melting process. This left branch of the processes is reasonably described by the simplified thermal model under the assumption of the linearity of laser light absorption. The right branch of the scheme shows the processes that are triggered by electron photoemission and cannot be taken into account by thermal modeling. Photoemission depletes a superficial target layer, causing the violation of the target quasineutrality. This results in a positive charging of the target as a whole and, thus, in the generation of an ambipolar electric field. The electric field forces the charge carriers to relocate in order to neutralize the excess positive charge. The rate of the neutralization process depends on a number of factors, which will be discussed below. All described processes were considered in the frames of the drift-diffusion approach (the LISC model), approving the possibility of Coulomb explosion in dielectrics under femtosecond-laser irradiation ${ }^{14,30,31}$ and showing such possibility for semiconductors under the action of nanosecond, UV laser pulses. ${ }^{31,32}$ By depleting the target layer of the hot electron population and causing electronic desorption/ ablation, photoemission can retard the heating and melting processes. Below we compare the results of simulations based on the thermal and LISC models in order to test the relevance of the latter and show its advantages.

\section{A. Thermal model}

The details of the thermal model used in this paper have been described in Refs. 25 and 46. Here we repeat only its main features, dwelling on the thorough calculation of the solid-liquid interface. ${ }^{25,53}$ The time-dependent temperature distribution $T(t, x)$ in the irradiated sample is governed by the heat-flow equation in a one-dimensional form as follows:

$$
\left[c_{p} \rho+L_{m} \delta\left(T-T_{m}\right)\right]\left(\frac{\partial T}{\partial t}-u(t) \frac{\partial T}{\partial x}\right)=\frac{\partial}{\partial x} \lambda \frac{\partial T}{\partial x}+\alpha_{\mathrm{ab}} I(x, t) .
$$

Here $\rho$ is the mass density of the target material, and $c_{p}$ and $\lambda$ are the thermal capacity and the thermal conductivity of the target material, respectively. The laser pulse of a Gaussian temporal profile $I(x, t)$ is attenuated exponentially along the target depth according to the Lambert-Beer law as follows:

$I(x, t)=(1-R) \frac{2 F_{0}}{\tau} \sqrt{\frac{\ln 2}{\pi}} \exp \left[-4 \ln 2\left(\frac{t}{\tau}\right)^{2}\right] \exp \left(-\alpha_{\mathrm{ab}} x\right)$,

where $F_{0}$ is the laser fluence, $\tau$ is the laser pulse duration (FWHM), $\alpha_{\mathrm{ab}}$ and $R$ are the absorption and reflection coefficients, respectively, whose temperature-dependent values are taken from Ref. 24, and $x$ is the distance from the target surface toward the bulk depth. The term $L_{m} \delta\left(T-T_{m}\right)\left(L_{m}\right.$ is the latent heat of fusion) allows performing through calculations of the liquid-solid interface, ${ }^{25,53}$ whose temperature is assumed to be continuous and equal to $T_{m}$. The $\delta$-function was approximated $\mathrm{as}^{53}$

$$
\delta\left(T-T_{m}, \Delta\right)=\frac{1}{\sqrt{2 \pi} \Delta} \exp \left[-\frac{\left(T-T_{m}\right)^{2}}{2 \Delta^{2}}\right],
$$

with $\Delta$ depending on the temperature gradient (the definitional domain of the $\delta$-function should be larger than three computational cells). $u(t)$ is the velocity of surface recession, which is defined under the assumption that the flow of vaporized material from the surface follows the Hertz-Knudsen equation and the vapor pressure above the vaporized surface can be estimated with the Clausius-Clapeyron equation as follows:

$$
u(t)=\beta \frac{p_{b}}{\rho}\left(\frac{m}{2 \pi k T_{s}}\right)^{1 / 2} \exp \left[\frac{L_{v}}{k}\left(\frac{1}{T_{b}}-\frac{1}{T_{s}}\right)\right] .
$$

Here the coefficient $\beta$ accounts for the back flux of the vaporized particles to the target surface. Since low laser fluences near melting and vaporization thresholds are considered here, we take $\beta=1$, assuming collisionless expansion of the ablation products. Also absorption of the laser irradiation by the laser-induced plasma plume is neglected. $L_{v}$ is the latent heat of vaporization, $k$ is the Boltzmann constant, and $T_{b}$ is the boiling temperature under a reference pressure $p_{b}$. The parameters $p_{b}$ and $T_{b}$ are taken only as normalizing values and do not assume boiling as the vaporization mechanism.

The temperature behavior at the target boundary is governed by the boundary condition assuming the possibility of thermal ablation:

$$
\left.\lambda \frac{\partial T}{\partial x}\right|_{x=0}=\rho u(t) L .
$$

When ablation is absent, this boundary condition is selfregulating to that accounting for the absence of heat flow through the target surface. Other conditions were as follows: 
$T(x, 0)=T_{0}$ and $T(\infty, t)=T_{0}$, where $T_{0}$ is the initial (room) temperature. Here we assume that the thermal wave does not reach the remote boundary during the calculation time. The used one-dimensional modeling is justified by the large irradiation spot size as compared to the thermally affected target layer over the computational time.

\section{B. Formulation of the LISC model for silicon irradiated by nanosecond, UV laser pulses}

Our aim here is to describe the electron dynamics in the laser-irradiated silicon targets under conditions of the violation of the quasineutrality caused by electron photoemission. This requires considering two interacting subsystems, electrons and lattice, while allowing charge separation in the irradiated sample. Hence, the model should include the continuity equations for holes and free electrons, the equations describing charge-carrier transport, and the Poisson equation to calculate the electric field resulting from the violation of the quasineutrality. As was mentioned above, such an approach was used to advantage in the analysis of conditions favorable to Coulomb explosion in dielectrics and semiconductors in different irradiation regimes, including femtosecond $^{14,30,31}$ and nanosecond ${ }^{31,32}$ laser pulses. As shown in Ref. 27, on a suprapicosecond time scale, the electron and lattice subsystems can be described in terms of their temperatures, $T_{e}$ and $T_{l}$, respectively. In Ref. 32, target heating was described using Eq. (2), implying $T_{e}=T_{l}$ as widely accepted for fairly long laser pulses of nanosecond time scale. Here we focus on the heating, melting, and ablation aspects, particularly on electronic ablation. Supposing a strong charge separation and the possibility of incomplete thermal equilibrium, we use two energy equations for the electron and lattice subsystems and follow the electronlattice relaxation process.

For a $193 \mathrm{~nm}$ laser wavelength (photon energy $\hbar \omega$ $=6.4 \mathrm{eV}$ ), the continuity equations describing charge-carrier generation in silicon (band gap energy $E_{g}=1.17 \mathrm{eV}$ ) include one-photon ionization and Auger recombination and is supplemented by a term describing the photoemission process as follows:

$$
\begin{aligned}
& \frac{\partial n_{e}}{\partial t}+\frac{1}{e} \frac{\partial J_{e}}{\partial x}=\alpha_{\mathrm{ab}} \frac{I(x, t)}{\hbar \omega} \frac{n_{a}}{n_{0}}+Q_{\mathrm{av}} n_{a}-\gamma n_{e}^{2} n_{h}-P E \frac{n_{a}}{n_{0}}, \\
& \frac{\partial n_{h}}{\partial t}+\frac{1}{|e|} \frac{\partial J_{h}}{\partial x}=\alpha_{\mathrm{ab}} \frac{I(x, t)}{\hbar \omega} \frac{n_{a}}{n_{0}}+Q_{\mathrm{av}} n_{a}-\gamma n_{e}^{2} n_{h} .
\end{aligned}
$$

Here $n$ and $J$ are the density and the electric current, respectively; indices $e, h$, and $a$ refer to electrons, holes, and neutral atoms; $n_{0}=n_{a}+n_{h} ; \gamma$ is the coefficient of Auger recombination, and $Q_{\mathrm{av}}$ is the temperature-dependent avalanche rate. ${ }^{27}$ Note that the one-photon ionization term is scaled for the available ionization centers in order to account for a possible saturation of ionization. To calculate the charge-carrier transport in the target, the electric currents $J_{e}$ and $J_{h}$ are written in the drift-diffusion form as ${ }^{54}$

$$
J_{e}=|e| n_{e} \mu_{e} E-e D_{e} \nabla n_{e}, \quad J_{h}=|e| n_{h} \mu_{h} E-e D_{h} \nabla n_{h} .
$$

The diffusion coefficients are calculated as $D_{e}=k T_{e} \mu_{e} / e$ and $D_{h}=k T_{e} \mu_{h} / e$, with $T_{e}$ representing the carrier temperature and $\mu_{e}$ and $\mu_{h}$ being the electron and hole mobilities, respectively. Here, for simplicity, we assume the mobilities of the electrons and holes in pure silicon to be constant and equal to the basic values $\left[\mu_{e}=0.15 \mathrm{~m}^{2} /(\mathrm{V} \mathrm{s})\right.$ and $\mu_{h}$ $=0.045 \mathrm{~m}^{2} /(\mathrm{V} \mathrm{s})$ (Ref. 55)]. In fact, the electron and hole mobilities in silicon are decreasing functions of both the temperature and the electric field, at least in the measured ranges (up to $600 \mathrm{~K}$ and $10^{6} \mathrm{~V} / \mathrm{m}$, respectively). ${ }^{54}$ However, under the conditions studied in this paper, when $T_{e}$ and $E$ values are typically much higher, the charge-transport parameters of silicon are poorly known. Test modeling with ten times reduced mobilities for the electrons and holes has not demonstrated a significant impact on the resulting charging and melting behaviors. Consequently, by emphasizing a qualitative picture of our modeling, we abandon speculating approximations of the mobilities and stay here at their basic values.

The electron photoemission term for UV laser irradiation, when photon energy well exceeds the sum of the band gap and the work function, can be written as

$$
\mathrm{PE}=\frac{1}{2} \alpha_{\mathrm{ab}} \frac{I(x, t)}{\hbar \omega} \exp \left(-\frac{x}{l_{\mathrm{PE}}}\right)
$$

where $l_{\mathrm{PE}}=12 \AA$ is the electron escape depth. ${ }^{56}$ Expression (10) implies that the generated electrons with the normal momentum component directed to the surface can escape into vacuum. Thus, by taking into account the equilibrium angular distribution for the carrier momenta established at nanosecond-laser irradiation conditions, ${ }^{31,32}$ we assume that, on average, half of the electrons produced through the onephoton ionization are immediately photoemitted from the surface region.

The photoemission and diffusion terms lead to charge separation in the target, resulting in electric field $(E)$ generation described by the following Poisson equation:

$$
\frac{\partial E}{\partial x}=\frac{e}{\varepsilon \varepsilon_{0}}\left(n_{h}-n_{e}\right),
$$

with a boundary condition defined by Gauss law. In Eq. (11), $\varepsilon$ is the dielectric permittivity of bulk silicon. In order to better satisfy the stability of the numerical procedure, Eqs. (9) and (11) are introduced into Eqs. (7) and (8), composing the final form of the continuity equations:

$$
\begin{gathered}
\frac{\partial n_{e}}{\partial t}-\mu_{e} n_{e} \frac{e}{\varepsilon \varepsilon_{0}}\left(n_{h}-n_{e}\right)-\mu_{e} E \frac{\partial n_{e}}{\partial x}-\frac{\partial}{\partial x} D_{e} \frac{\partial n_{e}}{\partial x} \\
=\alpha_{\mathrm{ab}} \frac{I(x, t)}{\hbar \omega} \frac{n_{a}}{n_{0}}+Q_{a v} n_{a}-\gamma n_{e}^{2} n_{h}-P E \frac{n_{a}}{n_{0}},
\end{gathered}
$$




$$
\begin{aligned}
\frac{\partial n_{h}}{\partial t} & +\mu_{h} n_{h} \frac{e}{\varepsilon \varepsilon_{0}}\left(n_{h}-n_{e}\right)+\mu_{h} E \frac{\partial n_{h}}{\partial x}-\frac{\partial}{\partial x} D_{h} \frac{\partial n_{h}}{\partial x} \\
= & \alpha_{\mathrm{ab}} \frac{I(x, t)}{\hbar \omega} \frac{n_{a}}{n_{0}}+Q_{a v} n_{a}-\gamma n_{e}^{2} n_{h} .
\end{aligned}
$$

Instead of the simplified expression (3) for light attenuation within the bulk, the Beer-Lambert equation was solved taking into account the depletion of the ground state during irradiation as follows:

$$
\frac{\partial}{\partial x} I(x, t)=-\alpha_{\mathrm{ab}} I(x, t) \frac{n_{a}}{n_{0}} .
$$

The energy equation for free electrons is written taking into account that the electrons generated through a onephoton ionization have an initial energy equal to the difference of the photon and band gap energies. The Auger recombination process results in heating the free-electron subsystem. Also it is assumed that the photoemitted electrons escape from the target, bringing away the average freeelectron energy. Hence, the final form of the electron energy equations is as follows:

$$
\frac{\partial E_{f}}{\partial t}=\left(\hbar \omega-E_{g}\right) \frac{\alpha_{\mathrm{ab}} I(x, t)}{\hbar \omega} \frac{n_{a}}{n_{0}}-E_{e} P E \frac{n_{a}}{n_{0}}+E_{g} \gamma n_{e}^{2} n_{h} .
$$

Here $E_{f}=n_{e} E_{e}$ is the energy density of the free-electron subsystem, with $E_{e}$ the average electron energy. The electron energy transport within the target is expressed in terms of the heat-flow equation as follows:

$$
A_{e}\left(\frac{\partial T_{e}}{\partial t}+\frac{J_{e}}{e n_{e}} \frac{\partial T_{e}}{\partial x}\right)=\frac{\partial}{\partial x} K_{e} \frac{\partial T_{e}}{\partial x}-g\left(T_{e}-T_{l}\right)+\Sigma(x, t),
$$

where $A_{e}=3 k / 2$ is the free-electron heat capacity, $K_{e}$ $=2 k^{2} \mu_{e} T_{e} / e$ is the thermal conductivity of the electrons, ${ }^{27} \mathrm{~g}$ is the electron-phonon coupling constant, and $\Sigma(x, t)$ is the source term, which has the following form: ${ }^{31}$

$$
\Sigma(x, t)=\frac{\partial E_{e}}{\partial t}=\frac{1}{n_{e}}\left(\frac{\partial E_{f}}{\partial t}-\frac{3}{2} k T_{e} \frac{\partial n_{e}}{\partial t}\right) .
$$

The heat-flow equation for the lattice is written as

$$
\left[c_{p} \rho+L_{m} \delta\left(T-T_{m}\right)\right] \frac{\partial T_{l}}{\partial t}=\frac{\partial}{\partial x} \lambda \frac{\partial T_{l}}{\partial x}+g\left(T_{e}-T_{l}\right) .
$$

The value $\tau_{l}=g / A_{e_{5}}$ is the hot carrier relaxation time and is taken to be $0.5 \mathrm{ps} .^{57}$ The surface recession velocity via the electronic ablation mechanism $u_{e}(t)$ will be introduced below in Sec. III D.

For solving the system of equations (10)-(18), we use an explicit numerical scheme on an irregular grid, dense in the near-surface absorption region (the numerical cells $\Delta x$ are $5 \AA$ wide) and rarefying toward the remote boundary with a factor of 1.01. At the remote boundary, the absence of the electron flow is allowed assuming a floating sample. The time step $\Delta t$ is selected empirically to better satisfy the numerical scheme stability and the approximation of the original equations.

\section{Analysis of surface charging}

A rough estimation of the electron photoemission yield through a unit surface area $\left(N_{\mathrm{PE}}\right)$ from the target of thickness $L$ can be obtained by the integration of the photoemission term (10) over time and space (the time moment $t=0$ corresponds to the center of the Gaussian profile of the laser beam) as follows:

$$
N_{\mathrm{PE}}=\int_{-\infty}^{\infty} \int_{0}^{L} \mathrm{PE} d t d x
$$

By substituting Eqs. (3) and (10) into Eq. (19) and neglecting the change in the reflection and absorption coefficients upon melting of the target surface, we have

$$
N_{\mathrm{PE}}=\frac{1}{2 \hbar \omega} \alpha_{\mathrm{ab}}(1-R) F_{0} \frac{\left\{1-\exp \left[-\left(\alpha_{\mathrm{ab}}+l_{\mathrm{PE}}^{-1}\right) L\right]\right\}}{\left(\alpha_{\mathrm{ab}}+l_{\mathrm{PE}}^{-1}\right)},
$$

or at $L \gg \alpha_{\mathrm{ab}}^{-1}$, which is our case,

$$
N_{\mathrm{PE}}=\frac{\alpha_{\mathrm{ab}}(1-R) F_{0}}{2 \hbar \omega\left(\alpha_{\mathrm{ab}}+l_{\mathrm{PE}}^{-1}\right)} .
$$

Under the assumption that there is no supply of electrons from the radiation-free sides of the target, the electric field generated on the target surface can be estimated by using Gauss law. The residual positive charge of the target is equal to the charge of electrons having left the target. For a plane target with the charge density $q(x)=-e\left[n_{h}(x)-n_{e}(x)\right]$ generated to the end of the laser pulse, this gives

$$
\left.E\right|_{x=0}=\frac{1}{2 \varepsilon \varepsilon_{0}} \int_{0}^{L} q(x) d x=\frac{e N_{I}}{2 \varepsilon \varepsilon_{0}}=\frac{e N_{\mathrm{PE}}}{2 \varepsilon \varepsilon_{0}},
$$

where $N_{I}$ is the number of excess positive ions (holes) accumulated in the target per unit surface area. Note that the value of the electric field in vacuum above the surface is $\varepsilon$ times higher than that in the target. Finally, we obtain an estimate of the maximum electric field generated on the surface of the unearthed silicon sample under the considered conditions:

$$
\left.E\right|_{x=0}=\frac{e \alpha_{\mathrm{ab}}(1-R) F_{0}}{4 \varepsilon \varepsilon_{0} \hbar \omega\left(\alpha_{\mathrm{ab}}+l_{\mathrm{PE}}^{-1}\right)} .
$$

The electric field that must be exceeded in order to break the atomic bonds (the critical field) can be found as ${ }^{31,32}$

$$
\left.E_{\mathrm{cr}}\right|_{x=0}=\sqrt{\frac{2 \Lambda_{\mathrm{at}} n_{0}}{\varepsilon \varepsilon_{0}}}
$$

where $n$ is the number density of the lattice and $\Lambda_{\text {at }}$ is the energy necessary to impart on an atom in order to remove it from the target. By using the latent heat of vaporization for silicon $\left[16115 \mathrm{~J} / \mathrm{g}\right.$ (Ref. 58)] to estimate the $\Lambda_{\text {at }}$ value, we obtain from Eq. (24) $E_{\mathrm{cr}} \approx 2.65 \times 10^{10} \mathrm{~V} / \mathrm{m}$. By comparing Eqs. (21), (22), and (24), we find the value of the critical laser fluence, starting from which nonthermal ion emission can be expected as a result of high surface charging: 


$$
F_{0 \mathrm{cr}}=\frac{4 \varepsilon \varepsilon_{0} \hbar \omega E_{\mathrm{cr}}\left(\alpha_{\mathrm{ab}}+l_{\mathrm{PE}}^{-1}\right)}{e \alpha_{\mathrm{ab}}(1-R)} .
$$

With $l_{\mathrm{PE}}=12 \AA$, we obtain $F_{0 \mathrm{cr}} \sim 0.15 \mathrm{~J} / \mathrm{cm}^{2}$. This value is only somewhat lower than that obtained in the experiments $\left(0.2 \mathrm{~J} / \mathrm{cm}^{2}\right)$. Expression (25) has been obtained for an ideal one-dimensional case, disregarding the electron and hole drift during the laser action. Electrons arriving at the surface layer in order to neutralize the excess positive charge should tend to increase the critical laser fluence. A more sophisticated picture of the processes can be obtained within the LISC model as described in Sec. III B.

The number of ions emitted from the target by the electrostatic force can be estimated by calculating the accumulated positive charge in excess of the value necessary for the generation of the critical electric field:

$$
N_{\text {exp }}=N_{I}-N_{\text {Ith }}=\frac{\alpha_{\mathrm{ab}}(1-R)\left(F_{0}-F_{0 \mathrm{cr}}\right) S_{R}}{2 \hbar \omega\left(\alpha_{\mathrm{ab}}+l_{\mathrm{PE}}^{-1}\right)},
$$

with $S_{R}$ being the irradiated spot area. Under our irradiation conditions $\left(S_{R}=0.5 \mathrm{~mm}^{2}\right)$, this gives

$$
N_{\exp } \sim 1.38 \times 10^{14}\left(F_{0}-F_{0 \mathrm{cr}}\right),
$$

where laser fluence is in $\mathrm{J} / \mathrm{cm}^{2}$. In Fig. 2, the nonthermal ion yield estimated from Eq. (27) with $F_{0 \mathrm{cr}}=0.2 \mathrm{~J} / \mathrm{cm}^{2}$ is presented by a solid line as a function of laser fluence, showing the same general tendency as obtained in the experiments.

\section{The rate of electronic ablation}

As soon as the critical electric field is reached in the surface target layer, positive ion ejection is initiated as an inevitable process for relieving the target of the electrostatic stress. At nanosecond-laser pulse regimes, a continuous electron photoemission can lead to a continuous ion emission in contrast to femtosecond irradiation regimes, where the electrostatic disintegration (called Coulomb explosion) can be considered as a synchronous effect for several superficial monolayers. ${ }^{14,30}$ Under nanosecond-laser irradiation, nonthermal ion emission is an extended process that lasts from the generation of the critical electric field until the partial neutralization of the excess positive charge by the electron supply from deeper target layers, or until laser pulse termination, or, for fluences above the melting threshold, until the melting moment since band gap collapse due to melting should lead to a rapid electron relocation and the screening of the electric field. Besides, melting gives an alternative mechanism of stress relieving by means of thermal vaporization, as described by Eq. (5). In the time interval from the generation of the critical electric field until target surface melting, the electric field at the surface does not exceed the critical value due to immediate ion emission or due to charge relocation. In terms of the LISC model formulated in Sec. III B and III C, the electronic emission rate $u_{e}(t)$ has to be introduced, which would prevent the electric field growth above the critical value.

The electronic rate of ablation can be written as $u_{e}$ $=\partial s / \partial t$, where $s$ is the momentary position of the target surface coinciding with the position $E=E_{\mathrm{cr}}$ (in the case of $E_{s}$

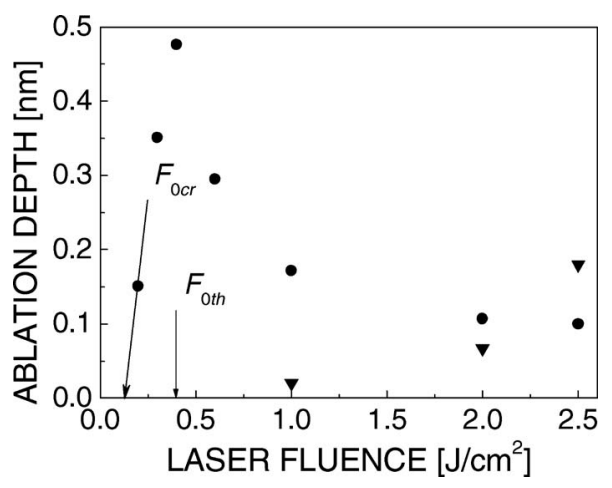

FIG. 7. Ablation depths obtained in the frames of the LISC model (circles) and the thermal model (triangles).

$\left.<E_{\mathrm{cr}}, u_{e}=0\right)$. For metal and semiconductor targets, the electric field generated in the sample decays quasiexponentially with the distance toward the target depth. ${ }^{31}$ Hence, we can assume that $E(x, t)=E_{s}(0, t) \exp \left(-x / x_{c}\right)$, where $E_{s}(0, t)$ and $x_{c}$ are the surface electric field and the characteristic length of its decay, respectively. For a rough numerical grid (here $\Delta x$ $=5 \AA$ ), the value $x_{c}$ can be evaluated from the calculated value of the electric field at the point $x=\Delta x$ as $x_{c}=$ $-\Delta x / \ln \left[E(\Delta x, t) / E_{s}(0, t)\right]$. We assume that, during the time step $\Delta t$, the surface electric field has increased from $E_{\mathrm{cr}}$ to $E_{s}$. Consequently, the position of the critical field (and, hence, a new position of the target surface) can be determined as

$$
x_{s}=\Delta x \frac{\ln \left[E_{\mathrm{cr}} / E_{s}(0, t)\right]}{\ln \left[E(\Delta x, t) / E_{s}(0, t)\right]} .
$$

Then, the final finite-difference form of the electronic ablation rate can be written as

$$
u_{e}(t)=\frac{\Delta x}{\Delta t} \frac{\ln \left[E_{\mathrm{cr}} / E_{s}(0, t)\right]}{\ln \left[E(\Delta x, t) / E_{s}(0, t)\right]} .
$$

Substituting the terms $\partial f / \partial t\left[\right.$ for $\left.f=\left(n_{e}, n_{h}, T_{e}, T_{l}, E_{f}\right)\right]$ by $\left[\partial f / \partial t-u_{e}(t) \partial f / \partial x\right]$ in Eqs. (12), (13), and (15)-(18), we obtain the LISC model with the target-vacuum interface moving due to the electronic mechanism of surface recession. Simulations have shown that, indeed, Eq. (29) provides for the fulfillment of the condition $E_{s}(0, t)=E_{\mathrm{cr}}$ as soon as the electronic ablation mechanism is realized.

\section{MODELING RESULTS AND DISCUSSION}

The simulations on the basis of the LISC model were performed for the laser fluence range of $0.2-2 \mathrm{~J} / \mathrm{cm}^{2}$. Assuming that the heated surface atoms having a certain vibrational energy can escape from the sample with a greater probability than the cold ones, a more correct expression for the critical field is used ${ }^{31}$

$$
\left.E_{\mathrm{cr}}\right|_{x=0}=\sqrt{\frac{2\left(\Lambda_{\mathrm{at}}-c_{p} T_{l}\right) n_{0}}{\varepsilon \varepsilon_{0}}} .
$$

The results of modeling are presented in Figs. 7-11. According to both models, the melting threshold fluence for silicon irradiated by the nanosecond-laser pulses (193 nm wavelength) is $\sim 0.4 \mathrm{~J} / \mathrm{cm}^{2}$ (Fig. 7), which is in good agreement with Ref. 24. The electronic model was applied only for a 

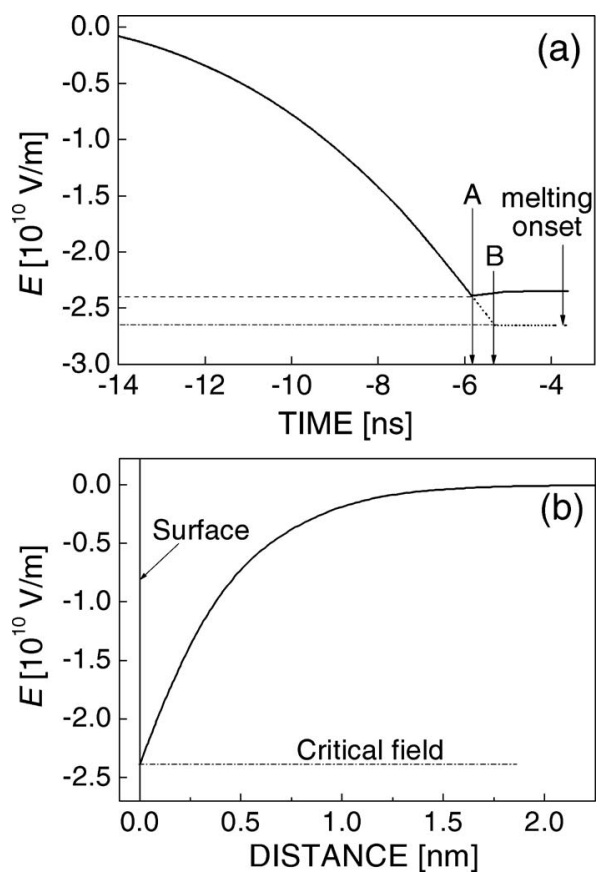

FIG. 8. (a) Calculated temporal evolution of the electric field generated on the surface of the silicon target irradiated by nanosecond, UV laser pulse at $1 \mathrm{~J} / \mathrm{cm}^{2}$ (solid line). The laser pulse is centered at $t=0$, while melting occurs at $t=-3.7 \mathrm{~ns}$. The critical electric field according to the temperaturedependent criterion given by Eq. (30) is reached at $-5.8 \mathrm{~ns}$ (shown by arrow A), whereupon the electronic ablation keeps it nearly constant. Neglecting the temperature dependence of the critical electric field [Eq. (24)] leads to a delay of the electronic ablation process (dotted line, arrow B). (b) Distribution of the electric field in the bulk at $t=-5.7 \mathrm{~ns}$ showing that the field is generated in a few superficial monolayers of the material.

time interval of the laser pulse action before reaching the melting temperature at the target surface. Already at $0.2 \mathrm{~J} / \mathrm{cm}^{2}$, which is well below the melting threshold, the electronic surface recession takes place, removing less than one monolayer of substance (Fig. 7) in accordance with the estimations presented in Sec. III C. The calculated threshold for electronic ablation $F_{0 \text { cr }}$ (shown in Fig. 7 by an arrow) is also in good agreement with the analytical estimate obtained in Sec. III C. At the melting threshold fluence $\left(0.4 \mathrm{~J} / \mathrm{cm}^{2}\right)$, the maximum of electronic ablation is reached when almost 2.5 monolayers of silicon $(4.8 \AA)$ are removed. At fluences above the melting threshold, the electronic ablation depth

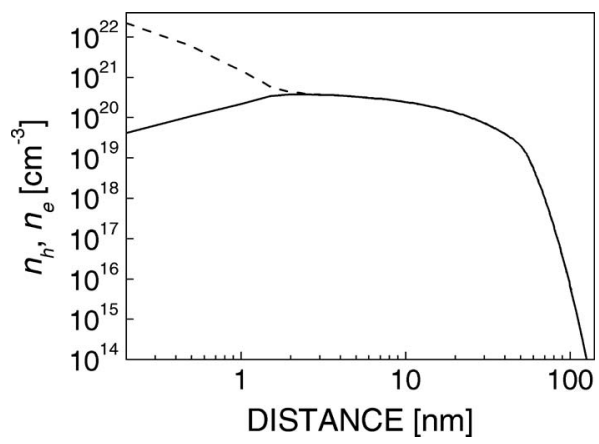

FIG. 9. Calculated spatial profiles of the electron (solid line) and hole (dashed line) densities in the Si target for the same conditions as in Fig. 8 $(t=-5.7 \mathrm{~ns})$. The strong electron depletion of the surface layer results in suppressed Auger recombination and, correspondingly, to extremely high surface charging.

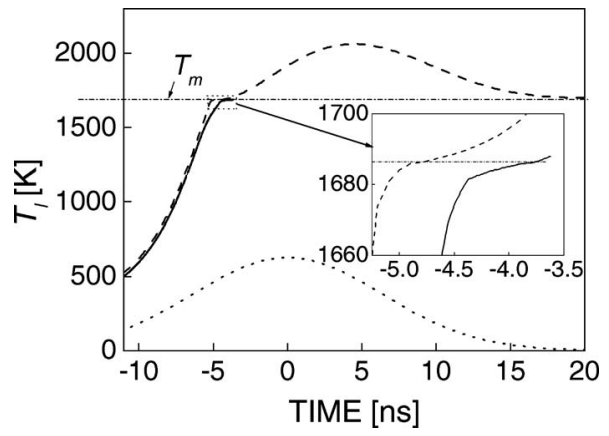

FIG. 10. Temporal evolution of the lattice temperature obtained within the frames of the LISC (solid line) and thermal (dashed line) models. The results on the LISC model are presented until the melting onset. The shape of the laser pulse is shown by the dotted line. The inset demonstrates details of the difference in the heating dynamics obtained with the two models in the vicinity of the melting point. The regime corresponds to Figs. 8 and 9 .

decreases, giving way to the thermal ablation mechanism. Consequently, at low fluences, only the nonthermal component of the ablation products is present while, with melting, the thermal component is superimposed on the nonthermal one (Fig. 7). Note that thermal ablation is weak at the studied fluence range. The thermal model shows that thermal vaporization with the removal of dozens to hundreds of monolayers develops only at fluences $\geqslant 3 \mathrm{~J} / \mathrm{cm}^{2}$, in agreement with our experimental observations, as reported in Fig. 5 (see Sec. II).

Thus, as a whole, the LISC model describes adequately the main features of electronic ablation observed in the experiments with the exception of the total ablation yield (cf. Figs. 2 and 7). Indeed, according to the simulations, the total ablation depth (thermal and electronic) decreases with fluence in the range of $0.4-1 \mathrm{~J} / \mathrm{cm}^{2}$, stabilizing at a low value
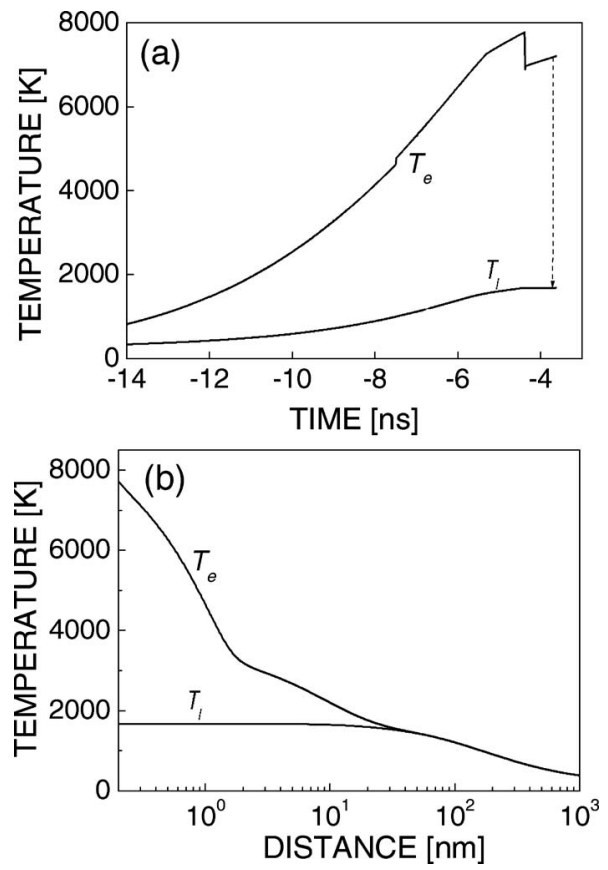

FIG. 11. (a) Temporal behavior and (b) spatial distribution at $t=-5 \mathrm{~ns}$ of the electron and lattice temperatures for the laser-irradiation regime as in Figs. $8-10$ showing the considerable nonequilibrium between the electron and lattice subsystems in the superficial target layer. 
for higher fluences until the thermal ablation mechanism becomes dominant at $F_{0}>2 \mathrm{~J} / \mathrm{cm}^{2}$, whereas the measured ion yield is a growing function of fluence. This can be attributed to the separate consideration of electronic and thermal ablations. We assume that electronic ablation terminates with the melting onset, and the generated electric field does not influence the thermal ablation process. However, it can be speculated that the positive charge accumulated on the sample surface before the melting onset further promotes the ablation process, leading to a combined ablation mechanism, chargeassisted thermal ablation. Note that the residual charge provides a strong, near-critical electric field [Eq. (30)]. The fact that the thermal model well describing the developed nanosecond regime of ablation overestimates the ablation threshold fluence is indicative of this charge-assisted mechanism of thermal ablation. ${ }^{59-61}$ A joint consideration of the two ablation mechanisms, electronic and thermal, which is extremely arduous, is beyond the limits of the proposed modeling approach. Below we will discuss what can be learned about the electron dynamics and the electric field generation in a laserirradiated silicon sample with the use of the LISC model and its comparison with the purely thermal model.

Figure 8(a) shows the temporal evolution of the electric field generated during the laser pulse with $F_{0}=1 \mathrm{~J} / \mathrm{cm}^{2}$ until the melting onset, which takes place at the time $t=-3.75 \mathrm{~ns}$ relative to the laser pulse maximum. Already before surface melting, the critical electric field has been reached in the surface layer (at $t=-5.8 \mathrm{~ns}$ ), giving way to the electronic mechanism of ablation [solid line in Fig. 8(a)]. Note that modeling with the electronic ablation criterion in the form (24) delays the ablation process by $0.5 \mathrm{~ns}$ as compared to the temperature-dependent $E_{\text {cr }}$ [Eq. (30)]. During the next $1.55 \mathrm{~ns}$ until the melting onset, almost one monolayer of silicon atoms (mainly in the form of positive ions as shown below) is emitted from the surface. The electric field is concentrated in a very narrow near-surface layer [Fig. 8(b), for $t=-3.8 \mathrm{~ns}$ ] with exponential decay toward the bulk, in accordance with a metallic behavior tending to accumulate the access charge on the surface. ${ }^{31}$ Indeed, Fig. 9 demonstrates that the excess positive charge is accumulated in a surface layer $\sim 2 \mathrm{~nm}$ wide. In this zone, a very high ionization degree is reached. The hole density on the surface is $n_{h} \approx 2.2$ $\times 10^{22} \mathrm{~cm}^{-3}$, which corresponds to the ratio $n_{h} / n_{0}=45 \%$, which is unusual for silicon at nanosecond irradiation regimes. It is widely believed that, under such regimes, the Auger recombination process regulates the free-electron density to a subcritical value. However, as discussed above, the superficial target layer is depleted of electrons due to photoemission, which results in the suppression of Auger recombination. Continuing the irradiation of the nanosecond-laser pulse leads to further ionization, which is not balanced by recombination. In turn, this leads to material softening and electronic surface recession.

It should be underlined that the described electronic mechanism of ablation/desorption at nanosecond irradiation regimes can be completely attributed to a short wavelength of laser irradiation that provides one-photon electron photoemission. For longer laser wavelengths leading to a multiphoton photoemission process, laser fluences near the melt- ing threshold cannot produce photoemission yields large enough for substantial target charging. Another feature of UV laser irradiation favoring the electronic mechanism of ablation is a small absorption length $(\sim 60 \AA$ for $\lambda$ $=193 \mathrm{~nm}$ ). The simulations show that, already at a depth of $\sim 0.2 \mu \mathrm{m}$ below the surface, the charge-carrier density corresponds to the intrinsic one $\left(1.5 \times 10^{10} \mathrm{~cm}^{-3}\right)$. For UV nanosecond-laser pulses, this provides favorable conditions for strong surface charging because of a negligible electronic supply from the bulk depth. It should be mentioned that, for UV femtosecond-laser pulses, the short absorption length leads to a strong localization of the laser energy in a thin target layer, resulting in extremely low melting thresholds, well below significant surface charging, and in phase explosion as the most probable ablation mechanism. ${ }^{31}$

It is interesting that the photoemission term scaled to the neutral atom density in Eqs. (12) and (15) assumes a selfregulation mechanism of photoemission at high values of the electric field. As soon as the electric field energy density reaches a value of the order of $1 \mathrm{eV} /$ atom and can noticeably influence the electron work function, the density of neutrals decreases, causing a decrease in the photoemission rate. As a result, the number of electrons that escape from the irradiation spot is saturated at overcritical laser fluences $\left(>0.2 \mathrm{~J} / \mathrm{cm}^{2}\right)$ and, for the irradiation spot size of $0.5 \mathrm{~mm}^{2}$ at $F_{0}=1 \mathrm{~J} / \mathrm{cm}^{2}$, the calculated photoelectron yield is $\sim 2.2$ $\times 10^{13}$, in excellent agreement with the measured electric residue (Fig. 3).

Figure 10 presents the lattice temperature behavior in the case of laser irradiation obtained by using the two models, thermal (dashed line) and LISC (solid line). The shape of the laser pulse is given by the dotted line. It is seen that the LISC model gives a very good approximation of the thermal model, showing only a slight lag in heating. This lag is attributed to a partial loss of the laser energy due to the photoemission and surface recession processes. Indeed, the calculations have shown that by setting $P E=0$ in Eqs. (12) and (15), we obtain exact concordance between the two models. Increased deviation between the temperatures obtained in the frames of two models occurs when the melting point is being approached. A comparison with Fig. 8(a) shows that the electric field reaches its critical value, giving rise to electronic ablation. The inset in Fig. 10 gives a more detailed difference between the results obtained, with the two models demonstrating a melting delay of $\sim 1 \mathrm{~ns}$ for the case when the photoemission process and electronic target recession are taken into account. An integration of the laser pulse shows that this seemingly weak delay actually corresponds to a substantial increase in laser energy coupled to the target until the melting onset exceeds $20 \%$ as compared to thermal modeling. This value is apparently smaller than that measured in the experiments (see Figs. 4 and 5); however, it shows that, indeed, the electronic mechanism of ablation of semiconductor targets can play a perceptible role in the overall balance of the laser energy.

It should be emphasized that the continuum approach used here can considerably underestimate the studied effects by disregarding the subtle surface processes such as the removal of adatoms and surface dimers, which have lower 
binding to the surface and can be removed by an electric field well below the critical one. Another issue concerns the strong concentration of holes in the surface layer, which is observed in Fig. 9 and points to the accumulation of antibonding states on the surface, which favor bond rupture and ion emission. ${ }^{11,62}$ These two factors can significantly contribute in increasing the laser energy necessary to produce surface melting and, thus, to further delay the melting moment during the laser pulse.

An important result obtained in the frames of the LISC model is the absence of equilibrium between the electron and lattice subsystems in the absorption zone. The temporal behavior of the electron and lattice temperatures at the surface and their spatial distributions are given in Fig. 11 for $F_{0}$ $=1 \mathrm{~J} / \mathrm{cm}^{2}$. It is seen that with heating time, the electron temperature deviates from that of the lattice, exceeding the latter by four to five times. One can notice sudden jumps in the electron temperature experienced during heating [Fig. 11(a)]. They can be attributed to the ultrafast dynamics of free electrons in the superstrong electric field. The electron diffusion, which is extremely weak in the depth of the order of several dozens of nanometers, can play, however, some role in the surface layer, where the electron temperature reaches values of 7000-8000 K. When reaching a value competitive with the drift term in Eq. (9), the diffusion term causes a sudden relocation to the surface of colder electrons from deeper target regions. After balancing between the drift and diffusion, the electron temperature increases until the melting moment. As soon as melting takes place, leading to the band gap collapse, the strong increase in the conduction band population should lead to a rapid equilibration between the electron and lattice subsystems, as shown by the arrow [Fig. 11(a)].

Figure 11(b) demonstrates that the nonequilibrium between the electrons and the lattice takes place in a layer $30 \mathrm{~nm}$ thick, where the main absorption of the laser light occurs. In deeper layers where the energy is transported via thermal conduction, the electron and lattice temperatures are equal. The enhanced electron temperature in the electron escape zone can also contribute to the increased electric field via the thermal emission mechanism. This is an additional point in favor of a significant effect of charge-carrier transport on the dynamic melting threshold in silicon under nanosecond-laser ablation reported in this study.

\section{CONCLUSIONS}

We have studied experimentally and theoretically an electronic mechanism of desorption and ablation of silicon under irradiation by nanosecond-laser pulses. Different aspects of the dynamics of laser-induced target heating, melting, and ablation have been analyzed, both thermal and electronic: laser light absorption, electron and lattice heat conduction, electron photoemission, generation of the ambipolar electric field, and charge-carrier transport within the target. The electronic ablation rate has been introduced into the LISC model, which has enabled describing the surface recession below the melting point. It has been shown that the electron photoemission process can play a significant role in overall target behavior, leading to an enhanced laser energy necessary to reach melting and to the violation of the thermal equilibrium between the electron and lattice subsystems. Further development of the model implies the introduction of a number of improvements such as thermal electron emission and a criterion for adatom emission. More features of the electron dynamics could be learned within a kinetic approach, which could enable considering the ballistic electrons. However, the present model has revealed an important role of the charge-carrier effect in nanosecond laserirradiated silicon and its conclusions can be extended to other semiconductor materials.

\section{ACKNOWLEDGMENTS}

The authors would like to thank A. V. Bulgakov and R. Kronquist for valuable discussion. Partial financial support from INTAS and SB RAS (INTAS Project No. 06-10000138949) and RFBR (Project No. 06-08-01196) are gratefully acknowledged.

${ }^{1}$ C. V. Shank, R. Yen, and C. Hirlimann, Phys. Rev. Lett. 51, 900 (1983).

${ }^{2}$ P. Stampfli and K. H. Bennemann, Phys. Rev. B 49, 7299 (1994).

${ }^{3}$ K. Sokolowski-Tinten, J. Bialkowski, and D. von der Linde, Phys. Rev. B 51, 14186 (1995).

${ }^{4}$ S. I. Kudryashov and V. I. Emel'yanov, JETP Lett. 73, 228 (2001).

${ }^{5}$ A. Rousse, C. Rischel, S. Fourmaux, I. Uschmann, S. Sebban, G. Grillon, $\mathrm{Ph}$. Balcou, E. Förster, J. P. Geindre, P. Audebert, J. C. Gauthier, and D. Hulin, Nature (London) 410, 65 (2001).

${ }^{6}$ J. Bonse, S. Baudach, J. Krüger, W. Kautek, and M. Lenzner, Appl. Phys. A: Mater. Sci. Process. 74, 19 (2002).

${ }^{7}$ J. Bonse, K.-W. Brzezinka, and A. J. Meixner, Appl. Surf. Sci. 221, 215 (2004)

${ }^{8}$ J. Eizenkop, I. Avrutsky, G. Auner, D. G. Georgiev, and V. Chaudhary, J. Appl. Phys. 101, 094301 (2007).

${ }^{9}$ O. Varlamova, F. Costache, J. Reif, and M. Bestehorn, Appl. Surf. Sci. 252, 4702 (2006).

${ }^{10}$ M. Guillermin, F. Garrelie, N. Sanner, E. Audouard, and H. Soder, Appl. Surf. Sci. 253, 8075 (2007).

${ }^{11}$ J. Kanasaki, A. Okano, K. Ishikawa, Y. Nakai, and N. Itoh, Nucl. Instrum. Methods Phys. Res. B 101, 93 (1995).

${ }^{12}$ R. Stoian, D. Ashkenasi, A. Rosenfeld, and E. E. B. Campbell, Phys. Rev. B 62, 13167 (2000).

${ }^{13}$ D. J. Lim, H. Ki, and J. Mazumder, J. Phys. D 39, 2624 (2006).

${ }^{14}$ R. Stoian, A. Rosenfeld, D. Ashkenasi, I. V. Hertel, N. M. Bulgakova, and E. E. B. Campbell, Phys. Rev. Lett. 88, 097603 (2002).

${ }^{15}$ M. Henyk, F. Costache, and J. Reif, Appl. Surf. Sci. 186, 381 (2002).

${ }^{16}$ S. Amoruso, G. Ausanio, R. Bruzzese, L. Gragnaniello, L. Lanotte, M. Vitiello, and X. Wang, Appl. Surf. Sci. 252, 4863 (2006).

${ }^{17}$ H. Dachraoui and W. Husinsky, Phys. Rev. Lett. 97, 107601 (2006).

${ }^{18}$ S. Paul, S. I. Kudryashov, K. Lyon, and S. D. Allen, J. Appl. Phys. 101, 043106 (2007)

${ }^{19}$ A. Kasuya and Y. Nishina, Phys. Rev. Lett. 57, 755 (1986).

${ }^{20}$ A. V. Bulgakov, I. Ozerov, and W. Marine, Thin Solid Films 453-454, 557 (2004).

${ }^{21}$ A. V. Bulgakov, I. Ozerov, and W. Marine, Appl. Phys. A: Mater. Sci. Process. 79, 1591 (2004).

${ }^{22}$ R. Stoian, A. Mermillod-Blondin, N. M. Bulgakova, A. Rosenfeld, I. V. Hertel, M. Spyridaki, E. Koudoumas, P. Tsanetakis, and C. Fotakis, Appl. Phys. Lett. 87, 124105 (2005).

${ }^{23}$ V. Hommes, M. Miclea, and R. Hergenröder, Appl. Surf. Sci. 252, 7449 (2006).

${ }^{24}$ S. De Unamuno and E. Fogarassy, Appl. Surf. Sci. 36, 1 (1989).

${ }^{25}$ N. M. Bulgakova, A. V. Bulgakov, and L. P. Babich, Appl. Phys. A: Mater. Sci. Process. 79, 1323 (2004).

${ }^{26}$ E. J. Yoffa, Phys. Rev. B 21, 2415 (1980)

${ }^{27}$ H. M. van Driel, Phys. Rev. B 35, 8166 (1987).

${ }^{28}$ T. Held, T. Kuhn, and G. Mahler, Phys. Rev. B 44, 12873 (1991).

${ }^{29}$ S. S. Mao, X.-L. Mao, R. Greif, and R. E. Russo, Appl. Surf. Sci. 127129, 206 (1998).

${ }^{30}$ N. M. Bulgakova, R. Stoian, A. Rosenfeld, I. V. Hertel, and E. E. B. 
Campbell, Phys. Rev. B 69, 054102 (2004).

${ }^{31}$ N. M. Bulgakova, R. Stoian, A. Rosenfeld, I. V. Hertel, W. Marine, and E. E. B. Campbell, Appl. Phys. A: Mater. Sci. Process. 81, 345 (2005).

${ }^{32}$ W. Marine, N. M. Bulgakova, L. Patrone, and I. Ozerov, Appl. Phys. A: Mater. Sci. Process. 79, 771 (2004).

${ }^{33}$ G. S. Higashi, Y. J. Chabal, G. W. Trucks, and K. Raghavachari, Appl. Phys. Lett. 56, 656 (1990).

${ }^{34}$ The melting threshold was determined from time resolved reflectivity measurements performed from the same targets under vacuum conditions. The measured value of $0.4 \mathrm{~J} / \mathrm{cm}^{2}$ is in good agreement with the one reported in Ref. 24. See also L. Patrone, Ph.D. thesis, University AixMarseille II, 2000.

${ }^{35}$ A. V. Bulgakov, W. Marine, O. F. Bobrenok, and I. Ozerov (unpublished).

${ }^{36}$ J. T. Dickinson, S. C. Langford, J.-J. Shin, and D. L. Doering, Phys. Rev. Lett. 73, 2630 (1994).

${ }^{37}$ F. Stietz, M. Stuke, J. Viereck, T. Wenzel, and F. Trager, Appl. Surf. Sci. 129, 64 (1998).

${ }^{38}$ W. Marine, M. Gerry, P. Thomsen-Schmidt, and J. M. Scotto d'Aniello, Appl. Surf. Sci. 69, 290 (1993).

${ }^{39}$ W. Marine and P. Mathiez, IEEE J. Quantum Electron. 22, 1404 (1986).

${ }^{40} \mathrm{~A}$ more complete analysis shows that further mechanisms such as spatial photon energy redistribution towards hot carriers (see Ref. 39) must be considered to account for such a DTM increase with laser fluence. The diffusion of these hot carriers deeper into the substrate, which results in volume heating of the lattice, tends to decrease the efficiency of surface heating.

${ }^{41}$ J. A. van Vechten, R. Tsu, and R. W. Saris, Phys. Lett. 74A, 422 (1979).

${ }^{42}$ B. Dragnea and B. Bourguignon, Phys. Rev. Lett. 82, 3085 (1999).

${ }^{43}$ F. Sanchez, J. L. Morenza, R. Aguiar, J. C. Delgado, and M. Varela, Appl. Phys. Lett. 69, 620 (1996).
${ }^{44}$ V. N. Tokarev, J. G. Lunney, W. Marine, and M. Sentis, J. Appl. Phys. 78, 1241 (1995).

${ }^{45}$ S. Fähler and H.-U. Krebs, Appl. Surf. Sci. 96-98, 61 (1996).

${ }^{46}$ N. M. Bulgakova and A. V. Bulgakov, Appl. Phys. A: Mater. Sci. Process. 73, 199 (2001).

${ }^{47}$ J. Neamtu, I. N. Mihailescu, C. Ristoscu, and J. Hermann, J. Appl. Phys. 86, 6098 (1999).

${ }^{48}$ A. V. Gusarov, A. G. Gnedovets, and I. Smurov, J. Appl. Phys. 88, 4352 (2000).

${ }^{49}$ T. E. Itina, J. Hermann, P. Delaporte, and M. Sentis, Phys. Rev. E 66, 066406 (2002)

${ }^{50}$ N. Y. Bykov, N. M. Bulgakova, A. V. Bulgakov, and G. A. Loukianov, Appl. Phys. A: Mater. Sci. Process. 79, 1097 (2004).

${ }^{51}$ Z. Zhang and G. Gogos, Phys. Rev. B 69, 235403 (2004).

${ }^{52}$ A. A. Morozov, Appl. Phys. A: Mater. Sci. Process. 79, 997 (2004).

${ }^{53}$ S. P. Zvavyi and G. D. Ivlev, Inzh.-Fiz. Zh. 69, 790 (1996) (in Russian).

${ }^{54}$ B. Van Zeghbroeck, Principles of Semiconductor Devices, 2004, http:// ece-www.colorado.edu/bart/book/

${ }^{55}$ R. E. Hummel, Electronic Properties of Materials (Springer-Verlag, Berlin, 1993).

${ }^{56}$ C. Sebenne, D. Bolmont, G. Guichar, and M. Balkanski, Phys. Rev. B 12, 3280 (1975).

${ }^{57}$ T. Sjodin, H. Petek, and H.-L. Dai, Phys. Rev. Lett. 81, 5664 (1998).

${ }^{58}$ Properties of Elements: Handbook, edited by M. E. Drits (Metallurgiya, Moscow, 1985) (in Russian).

${ }^{59}$ E. F. Lazneva, Laser Desorption (Izdatelstvo Leningradskogo Universiteta, Leningrad, 1990) (in Russian).

${ }^{60}$ E. F. Lazneva, Radiat. Eff. Defects Solids 115, 257 (1991).

${ }^{61}$ A. V. Bulgakov, personal communication (June 21, 2007).

${ }^{62}$ N. Itoh and A. M. Stoneham, J. Phys.: Condens. Matter 13, R489 (2001). 
Journal of Applied Physics is copyrighted by the American Institute of Physics (AIP). Redistribution of journal material is subject to the AIP online journal license and/or AIP copyright. For more information, see http://ojps.aip.org/japo/japcr/jsp 